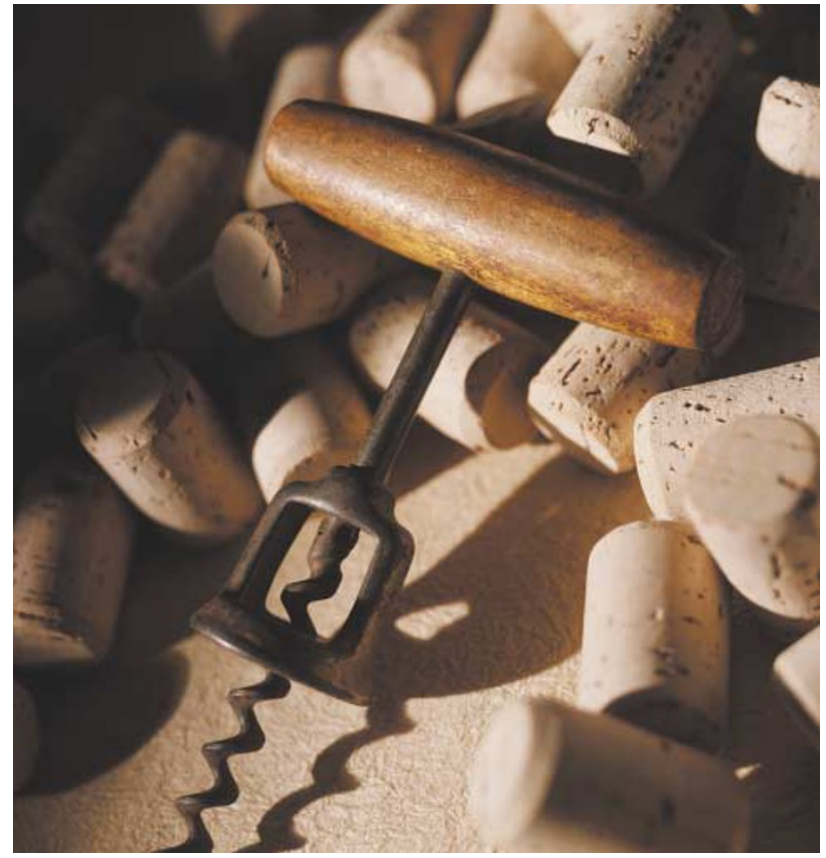

ION CHANNELS

\section{S4 opens another cork}

HCN channels are gated by hyperpolarization and cyclic nucleotides. As changes in membrane potential activate them, $\mathrm{HCN}$ channels must have a voltage sensor. In other voltageactivated channels, the fourth transmembrane domain of the protein - the S4 segment - fulfils this role. So, when exposed to voltage changes, the charged amino acids of $\mathrm{S} 4$ cause it to rotate and move outwards like a corkscrew, leading to channel opening. As HCN channels also have an $\mathrm{S} 4$ segment, this domain might also be the voltage sensor. However, the 'corkscrew' model accounts for the behaviour of depolarization-activated channels. How does the $\mathrm{S} 4$ segment of $\mathrm{HCN}$ channels behave in response to hyperpolarization? Data from Männikkö et al. indicate that S4 is indeed the voltage sensor of HCN channels, but that, in this case, the inward movement of $S 4$ leads to channel opening.

To explore whether S4 of HCN channels moves in response to voltage changes, the authors used the cysteine-accessibility method: they mutated the different residues of S4 to cysteines, and measured the accessibility of these cysteines to thiol reagents while the channel was open or closed. Using this method, it is possible to chart the positions of the residues with respect to the membrane and the solvent, and to outline their movement during channel activation. Männikkö et al. found that, similar to potassium channels (which are gated by depolarization), S4 moved in response to changes in membrane potential. But in this case, it was the inward movement of $\mathrm{S} 4$ in response to hyperpolarization that caused the channel to open.

So, the voltage-sensing mechanism is conserved in channels that are activated by voltage changes in opposite directions. As the actual channel gate seems to be near the intracellular side of the pore in both types of channel, we need to discover how voltage sensing is coupled to channel gating before we can understand how analogous movements of S4 lead to opposite effects on opening. Grasping how this coupling works is one of the great challenges in the field of ion channel function.

Juan Carlos López

6.) References and links

ORIGINAL RESEARCH PAPER Männikkö, R. et al. Voltage sensing mechanism is

conserved among ion channels gated by opposite voltages. Nature (in the press)

FURTHER READING Santoro, B. \& Tibbs, G. R. The HCN gene family: molecular basis of the

hyperpolarization-activated pacemaker channels. Ann. NY Acad. Sci. 868, 741-764 (1999)

NEUROLOGICAL DISORDERS

\title{
Lesions with a jagged edge
}

What is good for the embryo is not necessarily good for the adult, as a new report in Nature Medicine illustrates. In this paper, John et al. show that a signalling pathway that controls oligodendrocyte maturation in the embryo might contribute to the pathogenesis of multiple sclerosis (MS) if it is reactivated in the adult.

MS is an inflammatory disease that causes progressive demyelination in the central nervous system. This initially causes a deficit in axonal conduction, and unless remyelination occurs, the axons eventually degenerate because they lack the trophic support that myelin provides. In the early stages of the disease, the lesions are repaired quite efficiently, but the capacity for remyelination declines with time. However, even the most advanced lesions contain oligodendrocyte precursors that should be able to repair the damage, so why do they lose this ability in the later stages of MS?

The authors considered what other factors at the lesion site might be interfering with remyelination. The cytokine TGF- $\beta 1$ (transforming growth factor- $\beta 1$ ) is known to be present, and reactive astrocytes have also been implicated in the pathogenesis of MS. To examine how these components might interact to prevent remyelination, John et al. used microarray analysis to find out how TGF- $\beta 1$ affects the gene-expression profile of astrocytes in vitro.

One factor that was found to be upregulated in the presence of TGF- $\beta 1$ was a protein called jagged 1 . During normal development, jagged 1 acts as a ligand for Notch, which is expressed on the surface of immature oligodendrocytes. Binding of jagged 1 to Notch activates the expression of the basic helix-loop-helix transcription factor Hes5 in the oligodendrocyte precursors, and this prevents them from differentiating too early. The authors found that jagged 1 was expressed in active demyelinating lesions, but not in lesions in which remyelination was successful. This indicates that thejagged-Notch-Hes5 pathway is likely to be one of the factors that prevent the oligodendrocyte precursors in MS lesions from acquiring a mature myelinating phenotype.

Although TGF- $\beta 1$ is deleterious in terms of remyelination, blocking its activity altogether is not a viable option, because it also protects against inflammation. These new findings raise the possibility of intervention further downstream - for example, by interfering with Notch signalling - and this could lead to the development of new therapeutic strategies for the treatment of MS.

Heather Wood

(2) References and links ORIGINAL RESEARCH PAPER John, G. R. et al. Multiple sclerosis: re-expression of a developmental pathway that restricts oligodendrocyte maturation Nature Med. 8, 1115-1121 (2002) Future Med. 8, 1115-1121 (2002)
FURTHER READING Franklin, R. J. M. Why does remyelination fail in multiple sclerosis? Nature Rev. Neurosci. 3, 705-714 (2002)

\section{WEB SITES}

National Multiple Sclerosis Society:

http://www.nationalmssociety.org/

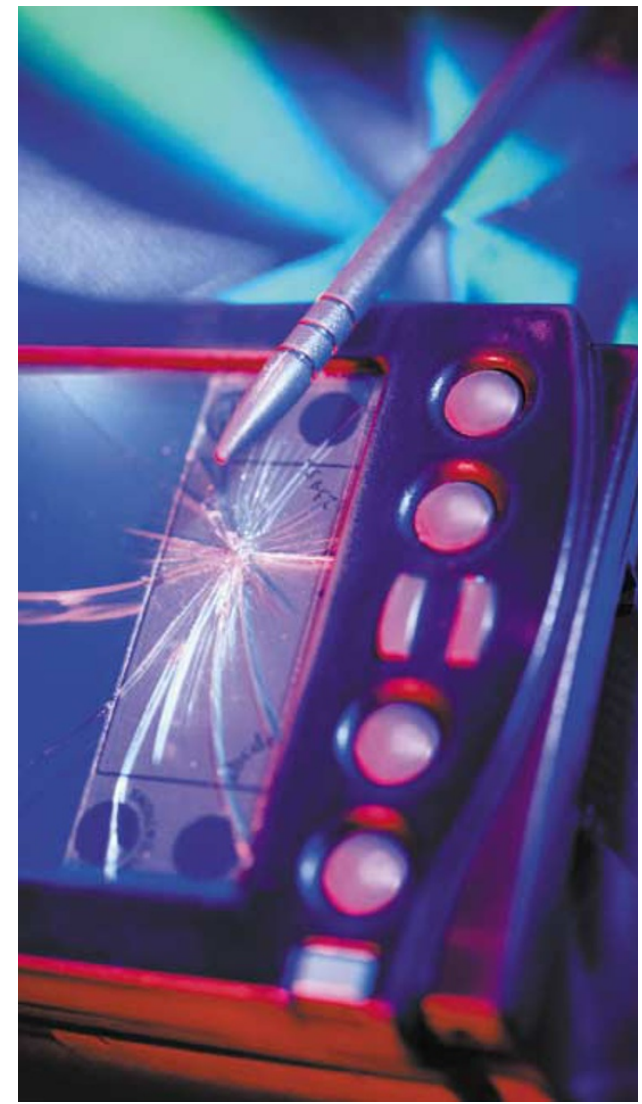

